\title{
ECG Based Integrated Mobile Tele medicine System for Emergency Health Tribulations
}

\author{
Ramesh Gamasu \\ M.Tech Research Scholar, Department of EEE, SACET, Cherala (A.P), India \\ ramesh.gamasu@gmail.com
}

\begin{abstract}
As health problem unpredictable in this polluted environment, it is better to keep an eye on our health. If a particular patient of heart or something dangerous disease then one should avail for the patient to monitor his health. It is not always possible to monitor him with at most attention. We should have a better system that always is with patient to monitor clearly and make the record of abnormalities obtained. As mobile phones commonly present in everybody pocket as a phone, player, pager, and so as our life saver, if we are in dangerous or unconscious condition, it alerts the user or alerts doctor or relative by making a call to family doctor or the some relative, who can care about the user by using telemedicine using mobile phone system. In this article, the alert system has a sensor to monitor the body condition like in case of heart. ECG is the best technique for monitoring and knowing the condition of the heart. And this sensor sends the data to the mobile phone through the Bluetooth wireless communication to eliminate the cumbersome cables and enables the absolute mobility. The mobile phone receives the biomedical signal and analyses the received signal with the reference signal and process the signal to get different types of information's that required to know the condition of the body. If any abnormalities like Tachycardia or Bradycardia (change of heart rate) in case of heart found in patient or users health then it will alert the patient by alarming and it make call to doctor in unconscious situations. The ECG graphical facilities are shown with help of Intel WML language.
\end{abstract}

Keywords: Bluetooth wireless communication, Cardiac Problems, ECG, Mobile

\section{Introduction}

In this hectic and polluted world taking care of health is necessary to live healthily particularly patients with heart problem. Cardiovascular disease is the world's leading killer, accounting for 16.7 million or 29.2 per cent of total global deaths. With modernization, a large proportion of young people are trading healthy traditional diets for fatty foods. Also most of them are employed with deskbound sloth nature of jobs that lacks any physical exercise. They also lead a stressful city life when compared to the relative calm of the countryside. These risk factors contribute to an alarming increase in high-risk cardiac patient population. Hence it becomes essential to monitor and alert the near ones of the patient and his family physician about the fatal condition that may occur at any instant [4]. Generally all monitoring systems are available for bedridden patients. All mobile telemedicine systems that have been designed so far have telemedicine systems that samples the medical data acquired, store it for a short duration and then transmits to the doctor end. That is they are available only for monitoring and do not alert the doctor or the near ones about the panic situation of the patient [11]. Different monitoring systems are commercially available and some of the 
research proposals about monitoring systems are classified in respect of the following features.

1. Systems that record signals and perform analysis off-line.

These systems only record the vital signals and no real time classification is done

2. Systems that perform remote real time monitoring.

Here the ECG signals are captured and sent to a monitoring centre through some communication media. The limitation here is that the analysis is not performed in the place where the signal is acquired.

3. Systems that provide local real-time classification.

These systems use intermediary local computers between sensors and the control centers or a hospital. These computers perform local real time monitoring. If some anomalies are detected, it sends alarms to the hospital. In those systems that make use of PCs to perform local real-time analysis, the mobility area of the patients is not very large [6]. It is almost reduced to their homes. Hence we propose a telemedicine system that monitors, performs real time analysis with the mobile phone and alerts the physician about the fatal condition. Also both the patient and the doctor can be mobile. This system maximizes the ability of cardiac patient to regain their independence and move freely [11].

\section{Telemedicine -'An Innovation in Communication Systems'}

Increasingly rapid advances in information and telecommunications technology are revolutionizing life and business around the world. The impact is being felt in the health sector with many new applications of these technologies. Telemedicine is essentially the use of both information technology and telecommunications to provide health services or support health service provision over a distance. The aim of this chapter is to outline the major new health uses of these technologies, what is this technology, describe the technologies, and provide a guide covering the key principles in this technology [20].

The following scenarios are designed to show the wide variety of possible applications using current standard technology

A hospital or clinic without an orthopedic surgeon uses a digital scanner and computer to relay an X-ray of a broken limb plus relevant history and examination findings to an orthopedic surgeon in a distant hospital to get advice on treatment and whether referral to the distant hospital for operative treatment is required. The provision of the $\mathrm{X}$-ray image allows much better advice than a phone discussion alone.

A small child is brought to a clinic after taking drugs prescribed for someone else. The clinic worker uses a computer to consult a drug information database for the appropriate course of action.

A doctor in a district hospital wants to maintain their level of professional expertise. By using a computer and the Internet (World Wide Web) the doctor is able to read the online versions of medical journals. Articles on particular topics are identified using online access to Medline.

Information technology can be an important tool for empowering people and enabling them to be more productive and effective in their work. It can also be a tool that exacerbates division and inequality by creating sections of the community which are information-rich and others that are information-poor. The aim of telemedicine developments should reflect this.

Telemedicine should always aim to support health workers providing care as close to the patients as possible [14]. This means information resources should be provided to all levels 
starting at primary care and clinical telemedicine should be used where possible to prevent referrals to district or tertiary hospitals and support care at the more local level. Used in this way, telemedicine can strengthen care at the primary care and district level.

Appropriate use of technology is all important. Telemedicine should be used to meet locally identified needs, and adequate technical support for users must be available. Telecommunication and computing technologies are becoming highly interdependent on each other and increasingly difficult to differentiate. This process is known as convergence and, together with telecommunication deregulation around the world, is resulting in advances in telecommunications technology as great if not greater than in computing [10].

\subsection{Local Real-Time Cardiac Telemedicine System}

The overview of the local real time monitoring system using mobile phone is show below. The system consists of four subsystems namely

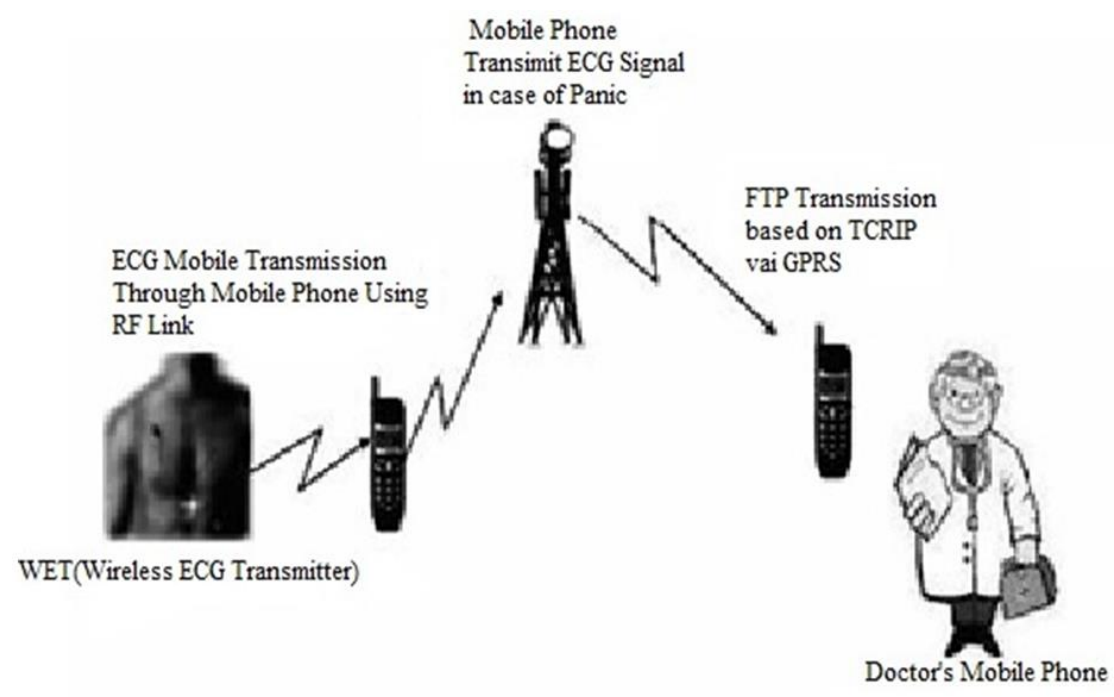

Figure 1. Telemedicine system

1. Signal such as ECG, blood pressure and body temperature are normally used to monitor a patient. Out of these signals we acquire only the ECG of the patient using miniature wearable chest equipment termed Wireless ECG Transmitter (WET). These signals are amplified, conditioned and transmitted to the mobile phone which is Bluetooth enabled.

2. The mobile phone has an in built ECG analyzer. It receives the ECG signal and processes it and calculates the heart rate of the patient in beats per minute (BPM).

3. This output in BPM is fed to a microcontroller. The ECG analyzer and microcontroller are designed to be present inside the mobile phone. The microcontroller declares panic situation in any of the following cases.

I. The beat per minute value is not between 60 and 100 .

II. Relatively fluctuating BPM for every heartbeat.

III. The R-amplitude falls below the minimum required by a healthy heart. Once the panic situation is declared by the microcontroller, the panic switch is 
closed and the automatic call function is triggered. Hence, the mobile phone establishes contact with the nearest medical center's server.

4. Only under panic condition, one minute sample of ECG signal of the patient is sent via the mobile network to the doctor using GPRS and an alerting SMS is given to the caretaker.

\subsection{Design of Wireless ECG Transmitter (WET)}

In simple terms, the WET is a wearable, miniature, chest equipment that is portable and fitted on the patient. The ECG electrodes acquire the ECG signals and instrumentation amplifier amplifies it. In order to make the processing easier the frequency of the signal is raised audio frequency range by a multiplier section. After analog to digital conversion, these ECG signals are transmitted to a mobile phone via Bluetooth Figure 2 illustrates the internal blocks of the WET. For the patients suffering from cardiac diseases it is very important to perform accurate and quick diagnosis. For this purpose a continuous monitoring of the ECG signal and the patient's current heart activity are necessary [9].

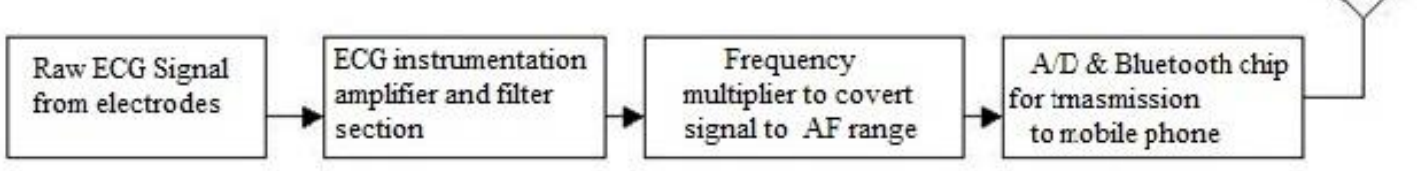

Figure 2. Essential blocks of the WET

The ECG electrodes continuously tap the ECG signal from the patient's body. The raw ECG signal's amplitude lies in the range of $0.1 \mathrm{mV}$ to $2 \mathrm{mV}$ that needs to be amplified for further processing. Its frequency range is between $0.5 \mathrm{~Hz}$ to $250 \mathrm{~Hz}$. The instrumentation amplifier serves as a pre-amplifier with a gain of 50. This pre-amplified signal is fed to a high pass filter with a cut off frequency of $0.5 \mathrm{~Hz}$. It is then followed by a post-amplifier of gain 10 and a low pass filter of cut off frequency of $250 \mathrm{~Hz}$. Hence, the high pass filter and the low pass filter together serve as a band pass filter limiting the frequency between $0.5 \mathrm{~Hz}$ to $250 \mathrm{~Hz}$. After pre and post amplification the signal is amplified 500 times. The amplified ECG signal lies in the extremely low frequency range $(0.5 \mathrm{~Hz}$ to $250 \mathrm{~Hz})$ [7]. Hence multiplying it to the audio frequency range will make processing and transmission of the signal easier. For instance, a PLL frequency multiplier can be utilized to accomplish such a task. PLL multipliers circuits operation is typical of all phase locked loops. Since the entire ECG signal ranges from $0.5 \mathrm{~Hz}$ to $250 \mathrm{~Hz}$, this frequency will be multiplied by a factor of 80 such that it is in the audio frequency range. Thus the multiplied signal will be in the range of $40 \mathrm{~Hz}$ to 20 $\mathrm{kHz}$. This audio frequency signal can be easily processed and transmitted. Short-Range Wireless (SRW) networks such as Bluetooth technology, RFID and IR, are gradually becoming more and more widespread in modern information systems. Most of these SRW networks have some drawbacks as the transmitting distance is less or else they are prone to disturbance due to outside environment $[13,17]$. The most feasible of them is, the Bluetooth as it has the essential ingredients whether it is the data rate or the error rate at which it transmits. Also studies indicate that Bluetooth technology is electro magnetically compatible with the tested medical devices. Hence we propose to use blue tooth link between the WET and ECG Analyzer. 
Blue tooth (IEEE 802.15) is a universal short range low power radio protocol operating in the unlicensed industrial, scientific and medical frequency band. It allows both data and voice transmission. The modulation technique is GSFK (Gaussian Frequency Shift Keying), with transmission at a rate of $1 \mathrm{M}$ symbols/s on one of 79 channels with $1 \mathrm{MHz}$ spacing in the $2.402 \mathrm{GHz}-2.480 \mathrm{GHz}$ band. Bluetooth uses the spread-spectrum frequency hopping connection with a rate of 1600 hops/s. Its key features are robustness, low complexity, low power and low cost. Compared to other methods of transmission, Bluetooth facilitates a noise free transmission. Moreover privacy and security options are highly advanced in the case of Bluetooth. We use BRC29 Blue Radios - 32 pin Bluetooth transceiver chip, which is a single chip solution for Bluetooth transmission. This chip requires an unregulated power supply of $1.9 \mathrm{~V}-3.4 \mathrm{~V}$ for its operation. Antennas need to be connected externally to the chip as shown in the Fig. 3. The transceiver chip serves both as a transmitter and a receiver. However, the mode of communication between the WET and the mobile phone is of the type simplex. Hence, the job of the Bluetooth transceiver situated in the WET is only to transmit. The transmitter starts with base band GFSK modulation data, which passes through a Gaussian filter. GFSK is a modulation technique where the data change the frequency of the carrier linearly for some amount of a carrier cycle during the duration of a bit [11]. The rate of frequency change is a function of the data rate. The amount of the frequency change is a function of the amplitude of the data. Hence, in this Pico-net, the WET plays the master and the mobile phone plays the slave.

\subsection{Mobile ECG Analyzer}

The analyzing software for the ECG signal is present inside the mobile phone. The transmitted ECG signal from the WET is received by the blue tooth enabled mobile phone, via Bluetooth link. The reverse process of the operations performed at the transmitting end, brings back the very same observed ECG signal. Hence, the operations like demodulation, D/A conversion and frequency division by a factor of 80 , have to be performed. Thus, the WET and mobile phone are synchronous with each other. This recovers back the originally observed ECG signal. The analyzer software checks the most significant parameter: the R-R interval of the PQRS waves used to find the BPM (beats per minute). To find the RR interval of the PQRS wave, the fact that the $\mathrm{R}$ wave is the most sharp, narrow and steep section of the wave, will be exploited. Moreover, the $\mathrm{R}$ section of the wave is the most sensitive one and any heart irregularity will instantly reflect on the $\mathrm{R}$ wave with great prominence [9].

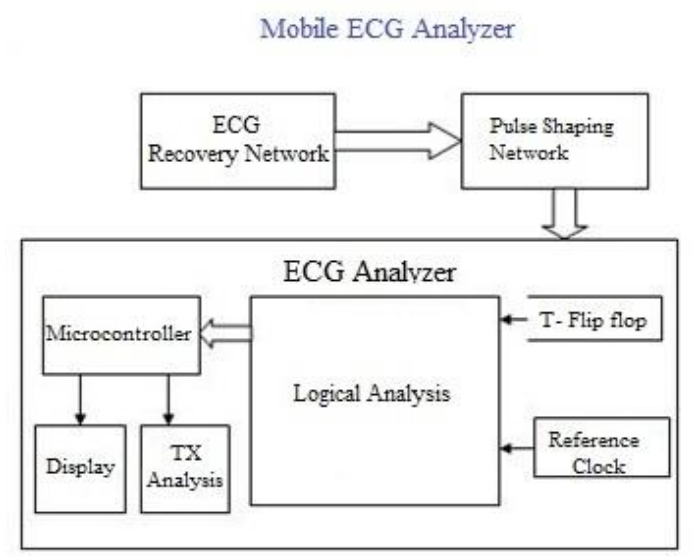

Figure 3. Essential blocks of ECG analyzer 
In order to measure the time interval between two $\mathrm{R}$ waves, focus is given on the upper halves of the $\mathrm{R}$ waves. Once the original ECG signal is recovered after demodulation and frequency division, it is fed to negative clipper software with positive $V_{\text {ref. }}$ The $V_{\text {ref }}$ is fixed at a value which is equal to the medically approved minimum $\mathrm{R}$ amplitude for a healthy heart. Consequently, a train of $R$ pulses above the zero axis by a amount of $+\mathrm{V}_{\text {ref }}$ is obtained. In order to coincide this train of pulses with the zero axes, it is necessary to clamp the signal by adding some voltage with negative $V_{\text {ref }}$. This gives a train of $R$ pulses based on zero voltage reference axes. This is fed to a software comparator whose output will be a train of pulses. These pulses can be counted by using counter. The no of give the no of R-waves. The counter output will be the number of clock pulses passed through the gate within an R-R interval. The microcontroller uses the below shown simple calculation to find the BPM (beats per minute). Where, $\mathrm{N}$ is the number of clock pulses counted by the respective counter. $1 \mathrm{~ms}$ is the time period of the clock pulse.

For example:

if $\mathrm{N}=800$

Then $\mathrm{BPM}=60 /\left(800^{*} 1 \mathrm{~ms}\right)=75 \mathrm{BPM}$,

This is a normal BPM value.

\subsection{Logical Analysis of ECG}

Any symptom of heart attack instantaneously reflects upon the R-R interval. Based on the ECG signal received the ECG analyzer calculates the heart rate in beats per minute. From this value obtained, analysis is done to verify whether the patient has a possibility of heart attack. The symptoms of heart attacks can be broadly classified into two types namely Tachycardia and Brady cardiac. Figure 4 shows the logic of the ECG analysis performed by the mobile ECG analyzer. When the BPM is above 100, it is said to be a tachycardia symptom. On the other hand, if the BPM falls below 60, it is said to be a bradycardia symptom [2]. Thus the $\mathrm{BPM}$ value for the normal sinus rhythm ranges between 60 and 100, i.e., normally the R-R interval should range between $600 \mathrm{~ms}$ and $1000 \mathrm{~ms}$.

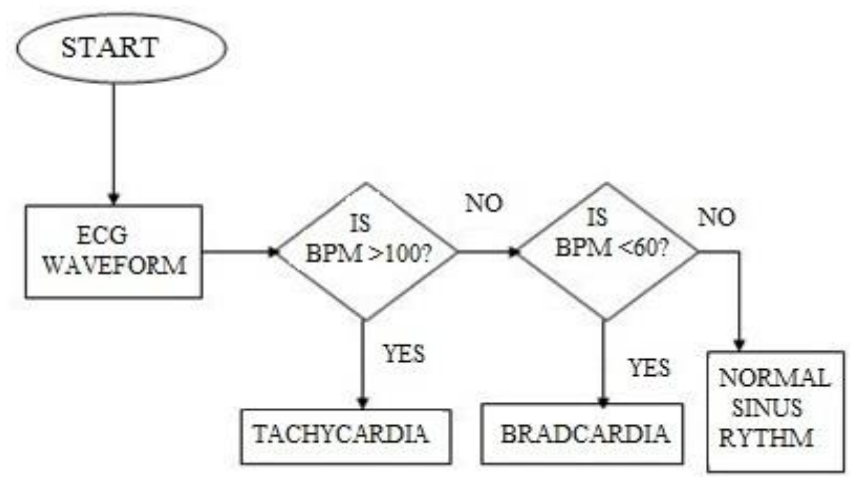

Figure 4. Flowchart showing the logic of ECG analysis

The mobile phone's microcontroller declares panic situation in any of the following cases:

1. The beat per minute value is not between 60 and 100 .

2. Relatively fluctuating BPM for every heartbeat. 
3. The R-amplitude falls below the minimum required by a healthy heart.

The mobile number of the doctor and the caretaker of the patient are stored in the microcontroller. Once the panic situation is declared by the microcontroller, the panic switch is closed and the automatic call function is triggered.The mobile phone establishes contact to the doctor by sending one-minute sample of ECG signal and an alerting SMS to the caretaker.

\subsection{Transmission of ECG Sample to Doctor}

Modern mobile communications are based on GSM and GPRS.GSM in standard mode of operation provides a data rate of $9.6 \mathrm{kbps}$. Whereas GPRS supports a data rate of $171.2 \mathrm{kbps}$ has performance superior to GSM. Further it has been shown that the method of using FTP over GPRS was by far superior to email. GPRS (General packet radio services) is a packet based wireless communication service that offers continuous connection, higher capacity mobile data services such as Internet/WAP, browsing email on the move, powerful visual communications, multimedia messages and location based services [1]. GPRS packet transmission offer a more user friendly billing than that offered by circuit switched services. Based on the above factors we proposed to send ECG transmissions using FTP via GPRS. Once the panic situation is declared and link has been established with the doctor's mobile phone using GPRS, the mobile phone of the patient transmits a one-minute sample of ECG signal via the mobile cellular network. The transmission of the ECG signal can be considered similar to the transmission of a digitized voice signal over a mobile cellular network [3].

\section{Present Work Done}

The software used here was Intel WML language to simulate the mobile telemedicine, now at a prototype stage has been tested for acquiring and transmitting the ECG signal to the ECG analyzer using simulation software. According to the software, the designed system was able to successfully transmit the ECG signals through Bluetooth dongle. For test purpose, test software has been used in the PC. By using this language we can make emulators of mobile phone. The emulator obtained here was working like mobile phone that simulates the present objective. The ECG received by the Bluetooth enabled emulator was given as input to the mobile emulator to analyze the data. The ECG analyzer was tested for both tachycardia and Bradycardia symptoms. The mobile phone successfully initiated a call function for both tachycardia and Bradycardia conditions. The Bluetooth link between the telemedicine system and mobile phone eliminates cumbersome cables and enables absolutely free mobility to the patient. Moreover, Bluetooth has high privacy and security features, which eliminates any chance of user collision and interference. Figure 5. is the emulator that used to simulate the telemedicine using mobile phone. The software we would use in original mobile phone is $\mathrm{j} 2 \mathrm{me}$. The Intel WML phone language software can also use directly into mobile phone to work for required objectives. The doctor's phone number would be stored in the user mobile phone so that it would make automatic call to the doctor when patient is in abnormal condition. And it also sends the data about the user, user's current location using GPS and biomedical information that got through physiological sensor.

To work this software first we give the address of the file that has to send to the mobile phone to analyze the signal (for the purpose of the simulation address has to be send, in actual Bluetooth sensor will automatically sends the data to mobile). The mobile phone emulator is shown below. It has all the facilities that a mobile phone should has like calls, SMS, MMS, GPRS, and all the facilities of mobile phone. 

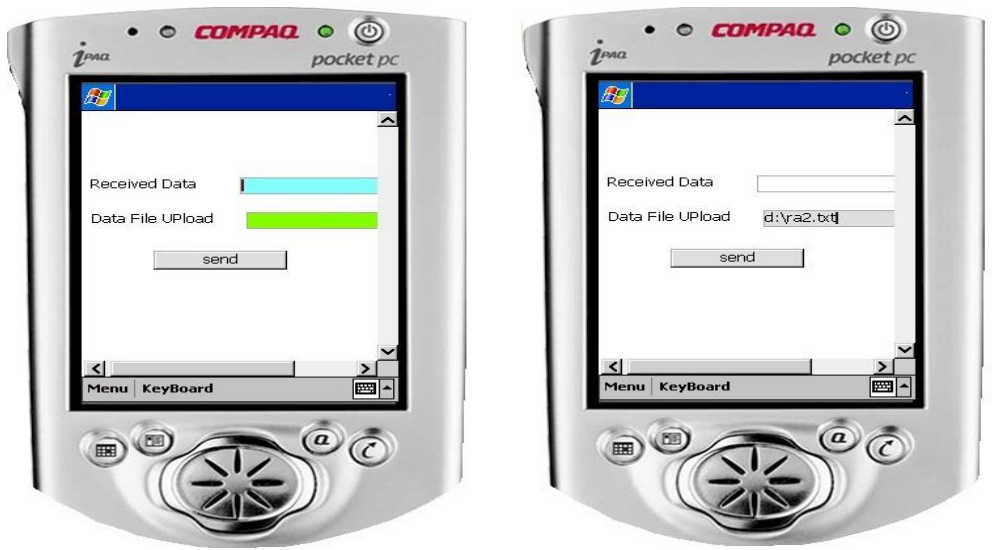

Figure 5. Bluetooth emulator (sensor part)

Here we use this mobile phone as data retriever from a Blue toothed sensor and data processor to analyze the data to get heart rate and some other heart parameters. The program here is like that if sensor emulator sends the signal it will get the address of the data and it will show the option to read the data or graph the data. By using read data option we can read the data like in single column matrix shown or otherwise it will make a graph of the given data.

And it has the options

1) ping: to know whether communication is there or not

2) Read file: to read or show the sampled values in a text format (it will give a column of data values that it received).

3) Draw graph: to draw the graph if user wishes to see.

The upload file browses the address of the file that has the data of ECG or other biomedical data. By pressing the send button the data will go to the mobile phone that is now another simulator. The mobile now got the data and now we can read the file by pressing the read file button. The mobile phone seems to be like below

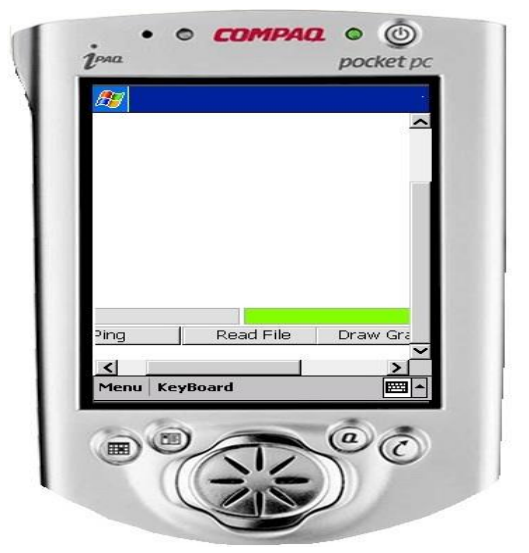

Figure 6. a) Mobile phone in normal mode

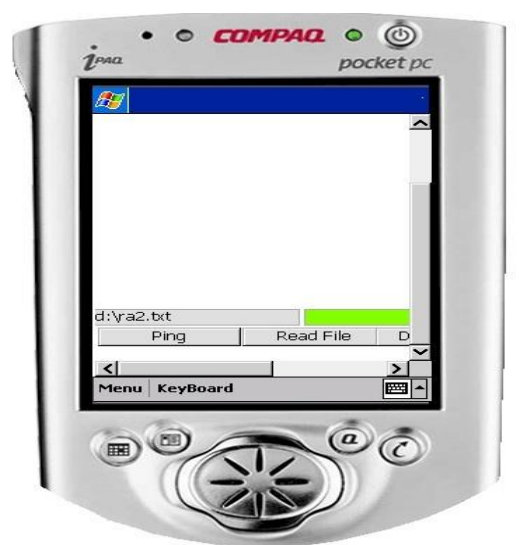

b) Phone retrieved address from sensor 

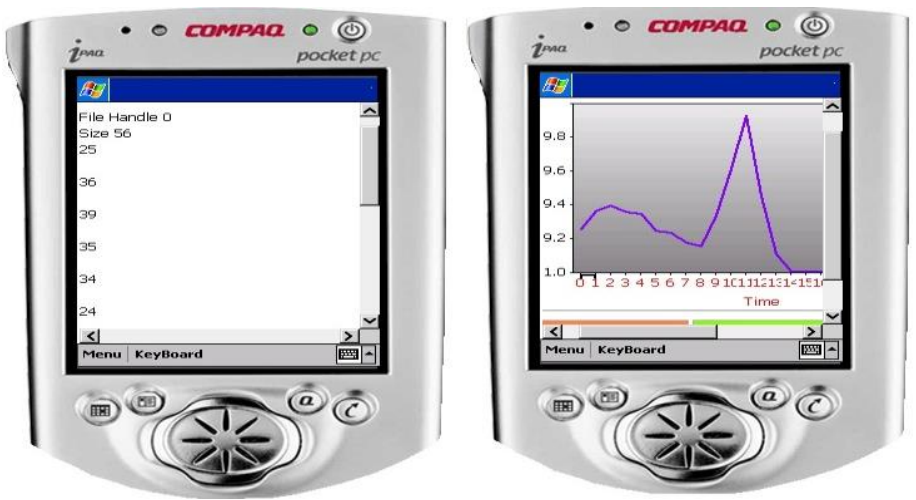

Figure 7. a) Mobile with read option selected. b) Mobile with draw graph option

The evolution of current mobile wireless communication and mobile network technologies will be the main driving force for the future developments in telemedicine. The developing mobile technologies like 3G wireless technology represent the convergence of various second-generation wireless systems, so that we can achieve more facilities. One of the most important aspects of $3 \mathrm{G}$ technology is its ability to unify existing mobile standards, such as CDMA, GMS, and TDMA under one umbrella.

3G mobile phone applications are found in various fields in the market but the possibilities for telemedicine are much higher than that of other areas. In the near future, the increasing medical data traffic and demand from different clinical applications and mobile medical sensors will be compatible with the data rates of current $3 \mathrm{G}$ systems. Specifically, in a society penetrated by $3 \mathrm{G}$ systems, home medical care and remote diagnosis will become common. Check-up by specialists and prescription of drugs will enable at home. In under-populated areas, virtual hospitals with no resident doctors will be realized based on high resolution transmission technologies and remote surgery. Also preventive medical care will be emphasized for individual health management. Medical data will be constantly transmitted to the hospital through built-in sensor and monitoring system and result will be fed back to the patients.

\section{Results and Discussions}

The complete mobile telemedicine, now at a prototype stage has been tested for acquiring and transmitting the ECG signal to the ECG analyzer using simulation software. According to the software, the designed system was able to successfully transmit the ECG signals through Bluetooth dongle. For test purpose, test software has been used in the PC.

The ECG received by the Bluetooth enabled PC was given as input to the simulated ECG analyzer. The ECG analyzer was tested for both tachycardia and Bradycardia symptoms. The mobile phone successfully initiated a call function for both tachycardia and Bradycardia conditions. The Bluetooth link between the system and mobile phone eliminates cumbersome cables and enables absolutely free mobility to the patient. Moreover, Bluetooth has high privacy and security features, which eliminates any chance of user collision and interference. The cellular phone manufacturing companies have been increased manufacturing the $3 \mathrm{G}$ mobile phones and service providers have also been entering into the $3 \mathrm{G}$ communication. It is good sign to make these types of applications so that we can get more telemedicine advantages. In this $3 \mathrm{G}$ service data transfer rate will be more and can get transfer of real time signals. 


\section{Future Aspects}

Mobile with some physiological sensor will give a better way of telemedicine such that we can get diagnosed by sitting in your home itself. The advancements of $3 \mathrm{G}$ technologies in mobile communications make the higher data transmission rates and advancements in IC technologies make the mobile processor more and more effective and powerful. With the Bluetooth technology we can combine the mobile and physiological sensor more and easier and can eliminate wires fully. User feel free without wires and it is comfortable.

Some of the combinations that can come in future more effectively are

\subsection{Mobile + ECG Electrodes}

This combination gives the tele-cardiology. More deaths are due to heart problems only. If we make this combination effectively we can get effective telemedicine system and we can get early detection of heart problems and can reduce the resulting heart failure deaths.

\subsection{Mobile + BMI Sensor}

This combination gives the better way to reduce the body cholesterol by early detection. It will give day by day weight and about how much you had in your lunch dinner and breakfast so that how many calories consumptions requires, all details can give by properly combining and programming the mobile phone.

\subsection{Mobile + EEG}

This combination gives the information about tension, problems in brain and main importantly the BCI (brain computer interface) used to control the outside world without making physical contact. This combination for the purpose of telemedicine may not that much useful but according to BCI it is more useful.

\subsection{Mobile + Camera}

This combination gives the effective way to the tele-dermatology. By making pictures of disease affected area and sending to the dermatologist gives better way of telemedicine. Now a day's all cellular phones coming with camera and MMS facility but nobody aware of this use.

\subsection{Mobile + Plethysmography}

This combination can give the volume changes in any limb so that we can find muscles working, blood changes, and different diseases. By making all these types of combinations we can get better way telemedicine and no need of going to the hospitals and consulting the doctors. In future all mobiles phones would be doctors having multi specialty degrees. These will cure the user disease by alerting, giving some prescriptions and or by consulting the specialist doctor through wireless communication. The coming technologies would have all these facilities.

\section{Conclusion}

Monitoring systems that perform a complete ECG analysis in a local device near the patients are of great interest because they allow us to improve the quality of life for those who suffer from cardiac disorders. For 'an anywhere at any time monitoring system, devices used 
have to be actually mobile. Hence, we recommend the usage of mobile phones as the core of these kinds of monitoring systems. In this dissertation, we have presented how mobile telemedicine system and ECG analyzer in the mobile phone, proves to be a life-saving tool for cardiac patients. As a future enhancement, we recommend the usage of GPS \& GIS for effective rescue of patient from any location by an ambulance in any case of cardiac emergency.

\section{References}

[1] T. Babineau and M. D. Ludman, "The Applications of Tele-health in Medical Genetics. In Tele-pediatrics: Telemedicine and Child Health", R. Wooten, and J. Batch, (eds.), Royal Society of Medicine Press, (2004), London, pp. 53-62.

[2] M. Caputo, "Telemedicine: linking patients and providers", University of Wisconsin Hospital, Pediatrics Grand Rounds, (2005), January 19.

[3] Center for Telemedicine Law, "Telemedicine licensure report", Office for the Advancement of Tele-health, HRSA, (2003).

[4] Center for Telemedicine Law. Telemedicine reimbursement report. Office for the Advancement for Telehealth, HRSA, (2003) June.

[5] M. A. Edwards, and A. C. Patel, "Telemedicine in the State of Maine: a model for growth driven by rural needs", Telemed J e-Health, vol. 9, (2003), pp. 25-39.

[6] J. J. Coelho, A. Arnold and J. Nayler, "An assessment of the efficacy of cancer genetic counseling using realtime videoconferencing technology (telemedicine) compared to face-to-face consultations", Eur J Cancer, vol. 41, (2005), pp. 2257-2261.

[7] J. Gray, K. Brain and R. Iredale, "A pilot study of telegenetics", Journal for Telemed Telecare, vol. 6, (2000), pp. 245-247.

[8] M. R. Gattas, J. C. MacMillan and I. Meinecke, "Telemedicine and clinical genetics establishing a successful service", Journal for Telemed Telecare, vol. 7, (2000), pp. 68-70

[9] D. Hailey, R. Roine and A. Ohinmaa, "Systematic review of evidence for the benefits of telemedicine", Journal for Telemed Telecare, vol. 8, no. S1, (2002), pp. 1-30.

[10] W. B. Karp, R. K. Grigsby and M. McSwiggan-Hardin, "Use of telemedicine for children with special health care needs", Pediatrics, vol. 105, (2000), pp. 843-847.

[11] D. H. Lea, J. L. Johnson and S. Ellingwood, "Telegenetics in Maine: Successful clinical and educational service delivery model developed from a 3-year pilot project", Genet Med., vol.7, (2005), pp. 21-27.

[12] T. E. Lobe, "Telemedicine and the future of healthcare for our children", Pediatrics, vol. 113, (2004), pp. 843847.

[13] J. P. Marcin, J. Ellis and R. Mawis, "Using telemedicine to provide pediatric subspecialty care to children with special health care needs in an underserved rural community", Pediatrics, vol. 113, (2004), pp. 1-6.

[14] J. A. Mitchel and G. Demiris, "Telegenetics: The next phase in the provision of genetics services", Genet Med., vol.7, (2005), pp. 1-2.

[15] P. S. Whitten, F. S. Mair and A. Haycox, "Systematic review of cost effectiveness studies of telemedicine interventions", BMJ, vol. 324, (2002), pp. 1434-1437.

[16] V. T. Bai and S. K. Srivastava, "Design and Simulation of Portable Telemedicine System for High Risk Cardiac Patients", Proceedings of world academy of science, engineering and technology, vol. 18, (2006), pp. 1307-6884.

[17] K. Yamauchi, W. Chen and D. Wei, "3G Mobile Phone Applications in Telemedicine -A Survey, The University of Aizu Fukushima of Japan, (1998), pp. 965-8580.

[18] S. Barro', J. Presedo D. Castro, M. Fernandez-Delgado, S. Fraga, M. Lama and J. Vil. "Intelligent Tele monitoring of Critical-Care Patients", IEEE Engineering in Medicine and Biology (1999), pp. 0739-51 IEEE July/Aug.

[19] P. Roncagliolo, L. Arredondo and A. Gonzalez, "Biomedical signal acquisition, processing and transmission using smart phone", $16^{\text {th }}$ Argentine Bioengineering Congress and Conference of Clinical Engineering IOP Publishing, Journal Of Physics, vol. 90, (2007), pp. 1-20. 


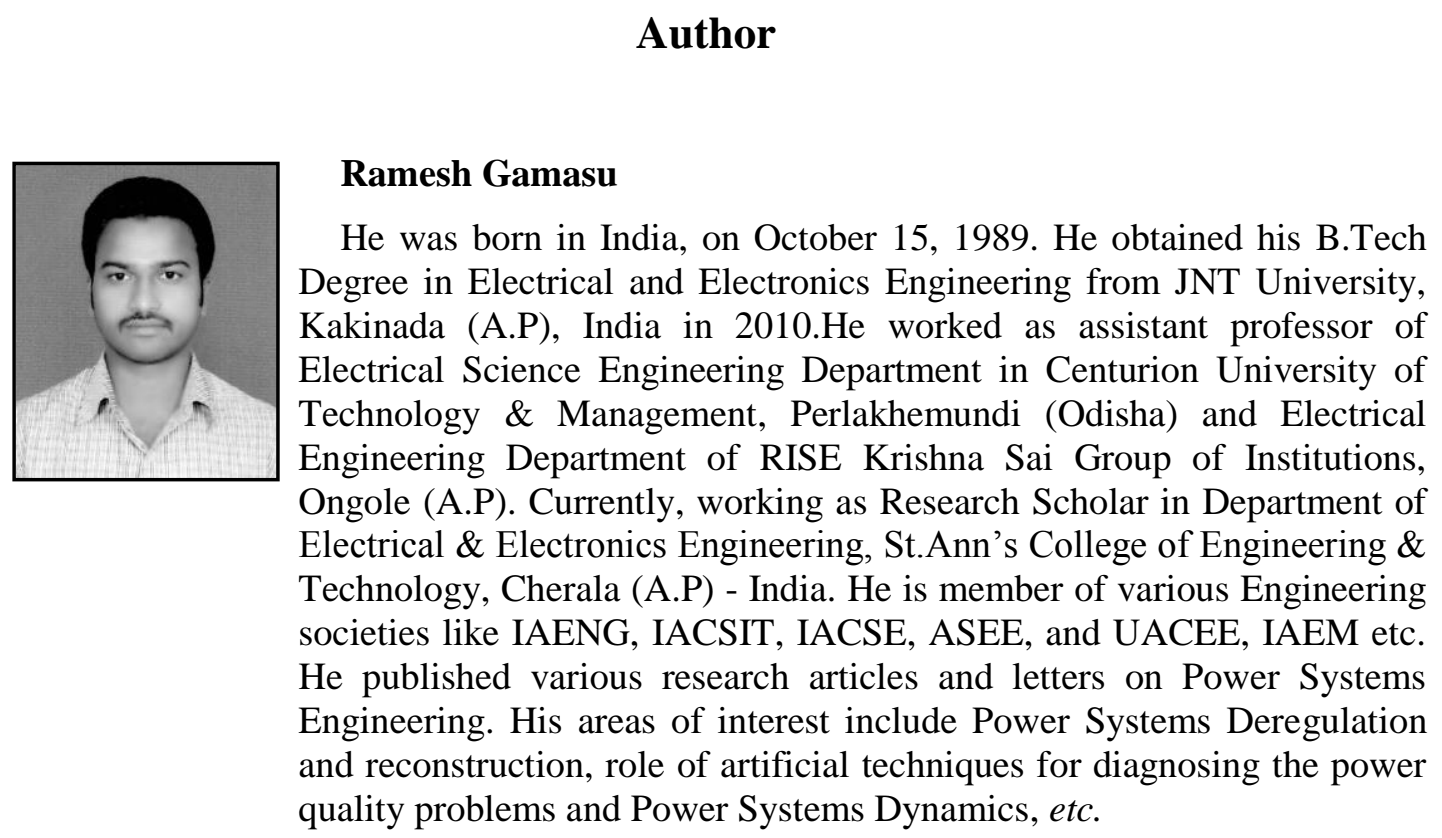

